\title{
Millimagnitude photometry for transiting extrasolar planetary candidates
}

\section{Follow-up of 30 OGLE transits. New candidates ${ }^{\star}, \star \star$}

\author{
P. Pietrukowicz ${ }^{1,2}$, D. Minniti ${ }^{1,3}$, R. F. Díaz ${ }^{4}$, J. M. Fernández ${ }^{1,5}$, M. Zoccali ${ }^{1}$, W. Gieren ${ }^{6}$, \\ G. Pietrzyński ${ }^{6,7}$, M. T. Ruíz ${ }^{8}$, A. Udalski ${ }^{7}$, T. Szeifert ${ }^{9}$, and M. Hempel ${ }^{1}$
}

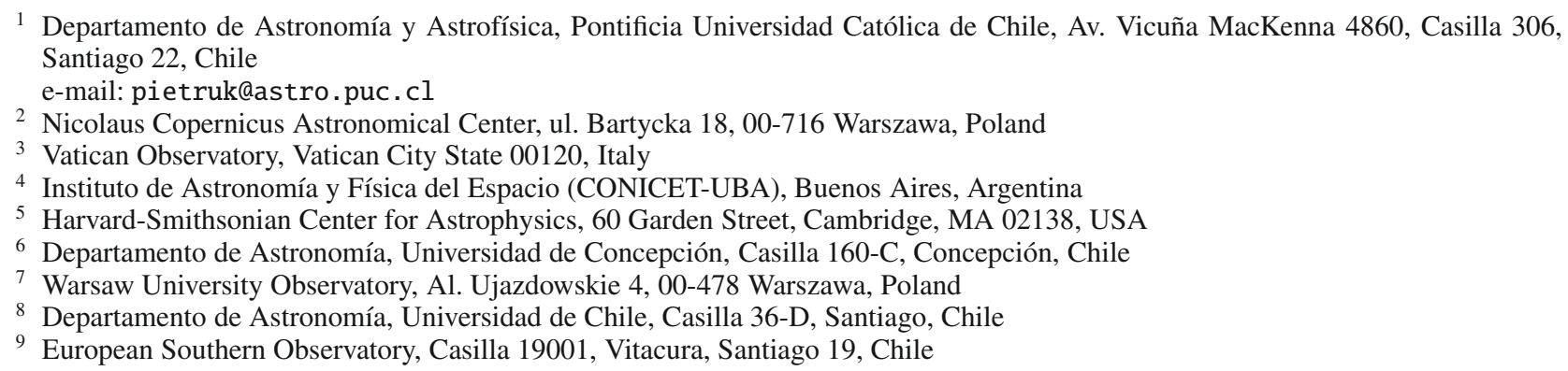

Received 24 March 2009 / Accepted 24 October 2009

\begin{abstract}
Aims. We used VLT/VIMOS images in the $V$ band to obtain light curves of the extrasolar planetary transits OGLE-TR-111 and OGLE-TR-113 and the candidate planetary transits: OGLE-TR-82, OGLE-TR-86, OGLE-TR-91, OGLE-TR-106, OGLE-TR-109, OGLE-TR-110, OGLE-TR-159, OGLE-TR-167, OGLE-TR-170, OGLE-TR-171.

Methods. Using difference imaging photometry, we were able to achieve millimagnitude errors in the individual data points. We present the analysis of the data and the light curves by measuring transit amplitudes and ephemerides and by calculating geometrical parameters for some of the systems.

Results. We observed nine OGLE objects at the predicted transit moments. Two other transits were shifted in time by a few hours. For another seven objects we expected to observe transits during the VIMOS run, but they were not detected.

Conclusions. The stars OGLE-TR-111 and OGLE-TR-113 are probably the only OGLE objects in the observed sample to host planets, with the other objects being very likely eclipsing binaries or multiple systems. In this paper we also report on four new transiting candidates which we have found in the data.
\end{abstract}

Key words. stars: individual: OGLE-TR-109, OGLE-TR-111, OGLE-TR-113 - planetary systems - binaries: eclipsing

\section{Introduction}

The field of extrasolar planets is developing rapidly, producing exciting results at an accelerated pace. The discovery of the first extrasolar "hot Jupiter" around the nearby solar-type star 51 Peg using precise radial velocity measurements (Mayor \& Queloz 1995) spurred a number of discoveries. Chief among these was the discovery of transits around the nearby solar-type star HD 209458 (Charbonneau et al. 2000; Henry et al. 2000). The success of the radial velocity studies also boosted extrasolar planetary searches using other techniques such as microlensing and transits. Currently more than 60 transiting extrasolar

\footnotetext{
* Based on observations collected with the Very Large Telescope at Paranal Observatory (ESO Programme 075.C-0427(A), DM and JMF visiting observers).

$\star \star$ Photometry of the transiting objects is available in electronic form at the CDS via anonymous ftp to

cdsarc.u-strasbg.fr $(130.79 .128 .5)$ or via

http://cdsweb.u-strasbg.fr/cgi-bin/qcat?J/A+A/509/A4
}

planets are known ${ }^{1}$. Many candidates were discovered by the OGLE team who carried out systematic searches, monitoring millions of stars along fields located in the Milky Way disk (Udalski et al. 2002a,b, 2003; Pont et al. 2008). Of more than 200 transiting candidates, already seven OGLE transits have been confirmed as being due to planets: OGLE-TR-10 (Konacki et al. 2005), OGLE-TR-56 (Konacki et al. 2003), OGLE-TR-111 (Pont et al. 2004), OGLE-TR-113 (Bouchy et al. 2004; Konacki et al. 2004), OGLE-TR-132 (Bouchy et al. 2004), OGLE-TR182 (Pont et al. 2008), OGLE-TR-211 (Udalski et al. 2008). Most of the other targets are eclipsing low-mass stars or brown dwarfs, or are due to blends of normal stars, triplets, etc.

Why such an interest in observing transiting candidates? The radial velocities give orbital parameters such as period, semimajor axis and projected mass $(M \sin i)$. The transits give not only the orbital parameters like period and inclination, but also the planet sizes: the eclipse amplitude is simply $(r / R)^{2}$. Thus,

\footnotetext{
1 See http://exoplanets.eu/
} 
Table 1. Basic information on the observed fields with VIMOS. Coordinates are given for the centers of the fields.

\begin{tabular}{clccccc}
\hline \hline $\begin{array}{c}\text { VIMOS field } \\
\text { with transit }\end{array}$ & $\begin{array}{l}\text { Short } \\
\text { name }\end{array}$ & $\begin{array}{c}\text { RA(2000.0) } \\
{[\mathrm{h}: \mathrm{m}: \mathrm{s}]}\end{array}$ & $\begin{array}{c}\operatorname{Dec}(2000.0) \\
{\left[{ }^{\circ}::^{\prime \prime}\right]}\end{array}$ & $\begin{array}{c}l \\
{\left[{ }^{\circ}\right]}\end{array}$ & $\begin{array}{c}b \\
{\left[{ }^{\circ}\right]}\end{array}$ & Duration of observations \\
\hline OGLE-TR-86 & F86 & $10: 58: 37.19$ & $-61: 31: 29.4$ & 289.905 & -1.540 & only first 8 h of night 1 \\
OGLE-TR-113 & F113 & $10: 52: 56.00$ & $-61: 28: 15.0$ & 289.269 & -1.783 & all four nights \\
OGLE-TR-167 & F167 & $13: 31: 36.00$ & $-64: 04: 15.0$ & 307.306 & -1.541 & $2 \mathrm{~h}$ of night 1 and all of night 2 \\
OGLE-TR-170 & F170 & $13: 14: 17.60$ & $-64: 44: 21.0$ & 305.368 & -1.976 & only nights 3 and 4 \\
\hline
\end{tabular}

from combined radial velocities and transits we know the mass of the planet without the inclination ambiguity and the radius, which in turn leads to a density. The few planets so studied appear to be indeed inflated gaseous planets, i.e. "hot Jupiters". One difficulty is that the derived planet radii are only reliable up to $10-15 \%$. More accurate transit photometry is needed to improve those estimates, as argued by Moutou et al. (2004). The discoveries by Pont et al. (2005a,b) of planet-sized stars around OGLE-TR-106 and OGLE-TR-122 for example, help to constrain the models of planetary systems. These planets are under intense irradiation, which inflates their sizes, depending on their own orbital parameters and intrinsic characteristics (Baraffe et al. 2003; Burrows et al. 2002). In some way the OGLE planets constitute the extreme cases, because of their very short periods.

We conducted a program to monitor photometrically the OGLE transit candidates, and we present here precise photometry for these transits. Some of the objects, namely OGLETR-109, OGLE-TR-111, OGLE-TR-113 have already been analyzed by Fernández et al. (2006), Minniti et al. (2007) and Díaz et al. (2007), respectively. In this paper we present an analysis of all OGLE transits in the VIMOS images. We have also searched for new transits.

Section 2 gives details on the observations, the selection and the properties of the sample. Section 3 describes the reductions of the data. In Sects. 4-8 we present the results obtained from the observed light curves of the OGLE transits. Section 9 describes the new transiting candidates we have found in the data. Finally, Sect. 10 states our main conclusions.

\section{The sample}

Our program was allocated 4 nights with VIMOS at the Unit Telescope 3 (UT3) of the European Southern Observatory Very Large Telescope (ESO VLT) at the Paranal Observatory during the nights of April 9 to 12, 2005. All four nights were clear throughout, with sub-arcsecond seeing during most of the time.

Before the run we prepared maps of the positions of the transit candidates in the OGLE fields and computed OGLE transit ephemerides. The selection of fields was based mostly on maximizing the number of interesting transiting candidates to be monitored given the VIMOS field of view. VIMOS is an imager and multi-object spectrograph (LeFevre et al. 2003). Its field of view consists of four $7^{\prime} \times 8^{\prime}$ fields covered by the four CCD chips arranged in a square pattern with a separation gap of about $2^{\prime}$. The CCD size is $2048 \times 2440$ pixels with a pixel size of $0{ }^{\prime} 205$.

We selected four fields in Carina which contain (but are not centered on) the following transit candidates: OGLE-TR-86, OGLE-TR-113, OGLE-TR-167, and OGLE-TR-170. We will refer to these fields as F86, F113, F167 and F170 respectively. Table 1 gives basic information on the monitored fields. In Fig. 1 we show the map of field F113 with the OGLE transit candidates included.

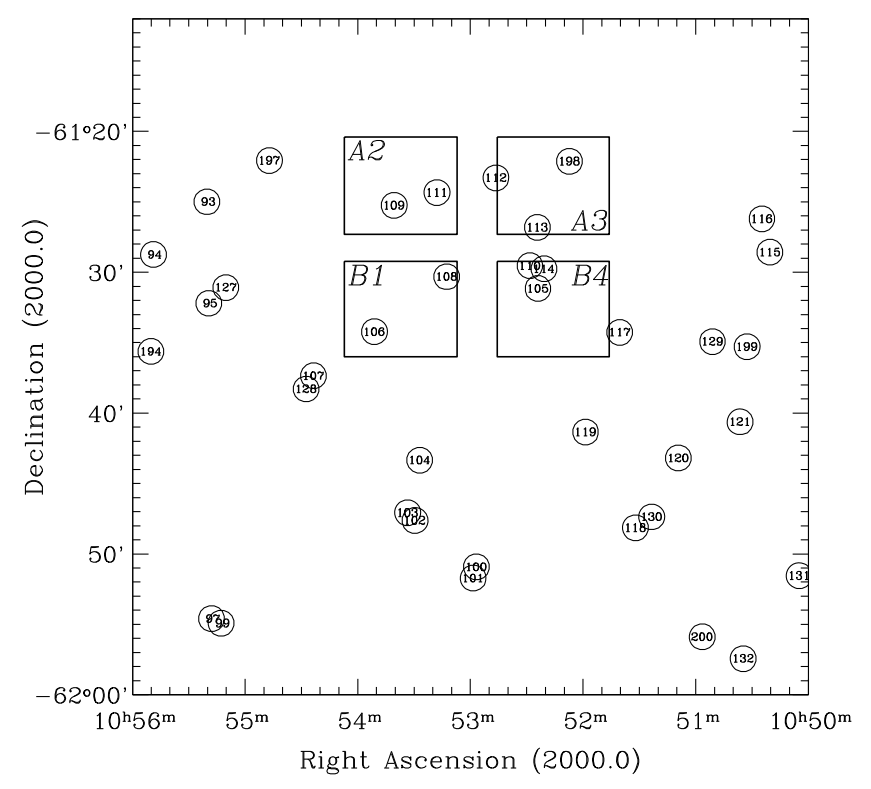

Fig. 1. Location of the OGLE transit candidates in the area of OGLETR-113 or the VIMOS field F113. VIMOS quadrants are marked and labeled as $A 2, A 3, B 1$ and $B 4$.

We monitored these four fields with a basic strategy to maximize the observing efficiency, trying to cover as many transit candidates as possible. Two fields per night were observed alternatively, with three $15 \mathrm{~s}$ images acquired per field before moving to the other field. With the help of the ESO Paranal staff, we reduced the nominal 9 min overhead between two different field exposures to $90 \mathrm{~s}$. Typically we obtained 150-300 images per night per field, resulting in well-sampled transits. All 32 OGLE transit candidates located in the VIMOS fields are listed in Table 2. This table indicates which candidates were expected to transit during our observations.

We observed with the $V$ filter only, since the sampling rate did not allow us to use two filters, and previous $I$-band observations are available from the OGLE database. One of the main objectives of this work was to discard blends and binary stars present among the transit candidates thanks to characteristic shape of transit events. The light curves measured in the $V$-band can also be compared with the I-band OGLE light curves. While the $I$-band is more efficient for transit searches (Pepper \& Gaudi 2005), the $V$-band shows the effects of limb darkening during the transit better and is more suitable for the modeling of the transit parameters.

\section{Photometry}

The bulk of the data acquired with VIMOS amounts to 82 GB. The periphery of the VIMOS images in each quadrant suffers from coma. Therefore, we cut a slightly smaller area for our 
P. Pietrukowicz et al.: Millimagnitude photometry of 30 OGLE transits. V.

Table 2. List of the OGLE transiting candidates in the VIMOS fields.

\begin{tabular}{|c|c|c|c|c|c|c|c|c|c|}
\hline $\begin{array}{l}\text { OGLE } \\
\text { transit }\end{array}$ & $\begin{array}{l}\text { VIMOS } \\
\text { field }\end{array}$ & $\begin{array}{c}P_{\text {OGLE }} \\
{[\mathrm{d}]}\end{array}$ & $\begin{array}{c}\text { Events } \\
\text { expected }\end{array}$ & $\begin{array}{l}\text { Were they } \\
\text { observed? }\end{array}$ & $\begin{array}{l}\text { OGLE } \\
\text { transit }\end{array}$ & $\begin{array}{l}\text { VIMOS } \\
\text { field }\end{array}$ & $\begin{array}{c}P_{\mathrm{OGLE}} \\
{[\mathrm{d}]}\end{array}$ & $\begin{array}{c}\text { Events } \\
\text { expected }\end{array}$ & $\begin{array}{l}\text { Were they } \\
\text { observed? }\end{array}$ \\
\hline TR-81 & F86 & $3.2165(6)$ & 0 & - & TR-111 & F113 & $4.0161(8)$ & 1 & fully \\
\hline TR-82 & F86 & $0.76416(14)$ & 0 & sinusoidal var & TR-112 & F113 & $3.8790(8)$ & 1 & star is saturated \\
\hline TR-83 & F86 & $1.5992(3)$ & 0 & - & TR-113 & F113 & $1.4325(3)$ & 1 & fully \\
\hline TR-84 & F86 & $3.1130(6)$ & 1 & no & TR-114 & F113 & $1.7121(3)$ & 1 & ?, bad seeing \\
\hline TR-85 & F86 & $2.1146(4)$ & 1 & no & TR-198 & F113 & $13.631(3)$ & 0 & - \\
\hline TR-86 & F86 & $2.7770(6)$ & 1 & partially & TR-159 & F167 & $2.1268(4)$ & 1 & fully \\
\hline TR-87 & F86 & $6.6067(13)$ & 0 & - & TR-160 & F167 & $4.9018(10)$ & 0 & - \\
\hline TR-88 & F86 & $1.2501(3)$ & 0 & star is saturated & TR-161 & F167 & $2.7473(5)$ & 0 & - \\
\hline TR-91 & F86 & $1.5790(3)$ & 1 & partially & TR-162 & F167 & $3.7582(7)$ & 1 & no \\
\hline TR-126 & F86 & $5.1108(10)$ & 0 & - & TR-163 & F167 & $0.94621(18)$ & 0 & - \\
\hline TR-192 & F86 & $5.4239(11)$ & 0 & - & TR-164 & F167 & $2.6815(5)$ & 0 & - \\
\hline TR-105 & F113 & $3.0581(6)$ & 0 & - & TR-166 & F167 & $5.2192(10)$ & 0 & - \\
\hline TR-106 & F113 & $2.5358(5)$ & 1 & fully & TR-167 & F167 & $5.2610(10)$ & 1 & partially \\
\hline TR-108 & F113 & $4.1859(8)$ & 1 & no & TR-170 & F170 & $4.1368(8)$ & 1 & fully \\
\hline TR-109 & F113 & $0.58909(12)$ & 4 & 3 events & TR-171 & F170 & $2.0918(4)$ & 1 & fully \\
\hline TR-110 & F113 & $2.8486(6)$ & 1 & partially & TR-172 & F170 & $1.7932(4)$ & 1 & no \\
\hline
\end{tabular}

analysis of $1900 \times 2100$ pixels, covering 7! $18 \times 6$ 6.49. The photometry was extracted with the help of the Difference Image Analysis Package (DIAPL) ${ }^{2}$ written by Woźniak (2000) and recently modified by Pych. The package is an implementation of the method developed by Alard \& Lupton (1998). To get a better quality of the photometry we worked on $475 \times 525$ pixel subfields.

Reference frames were constructed by combining the $8-13$ best individual images (depending on the field and the quadrant). Profile photometry for the reference frame was extracted with DAOPHOT/ALLSTAR (Stetson 1987). These measurements were used to transform the light curves from differential flux units into instrumental magnitudes, which later were transformed into the standard $V$-band magnitudes by adding an offset derived from $V$-band magnitudes of the transits in the fields (Díaz et al. 2007; Minniti et al. 2007).

The $15 \mathrm{~s}$ exposure times saturate stars at $V \approx 15.4 \mathrm{mag}$ in the images under the best seeing. This affected two transit candidates: OGLE-TR-88 and OGLE-TR-112.

The brightest planetary transit candidate monitored here is OGLE-TR-109, with $I=14.99$ and $V=15.82 \mathrm{mag}$, for which photometry with $\sigma_{V}=0.002 \mathrm{mag}$ was obtained. The faintest candidate is OGLE-TR-108, with $I=17.28$ and $V=18.73 \mathrm{mag}$ at $\sigma_{V}=0.009 \mathrm{mag}$.

Due to the coma the photometric quality at the extreme corners of the VIMOS fields is degraded. This affects the data for OGLE-TR-126 and to a minor degree for OGLE-TR-87 and OGLE-TR-108.

\section{Results}

Twenty-one transits were expected for 18 OGLE stars during the observations. Figure 2 illustrates the transit times calculated for the candidates in the field F113. For the object OGLE-TR109 with the short period of $P=0.589127 \mathrm{~d}$, four transits were expected. The object OGLE-TR-112 is saturated and no photometry was obtained in this case. In total, we observed thirteen events in 11 stars. Six transits were not detected and one is under question, due to bad seeing at the end of night four.

2 The package is available at http://users. camk. edu.pl/pych/ DIAPL/

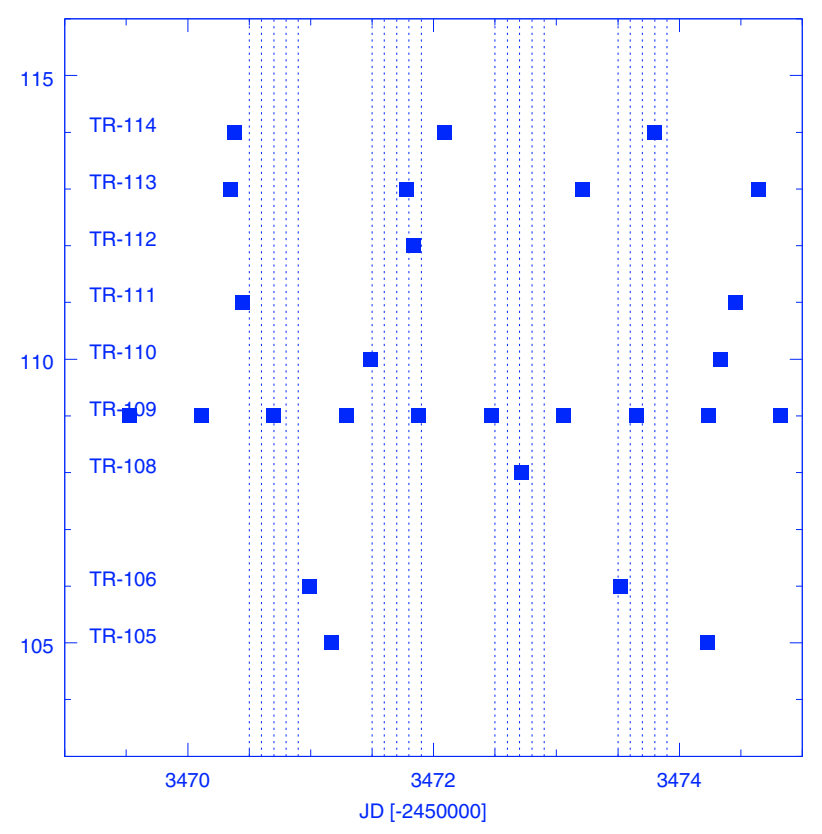

Fig. 2. Computed transit times for nine OGLE candidates in the field F113.

For 14 stars no transits were expected. However, even flat light curves are useful to examine possible light curve modulations due to ellipsoidal variability (Drake et al. 2003; Sirko \& Paczyński 2007).

Table 2 also indicates whether we observed a full transit, a partial transit, no transit or sinusoidal variations. The relatively small number of expected and detected transits in the fields F86 and F167 is the result of the short period of observation of these two fields. The highest efficiency is for F113, which is the only field observed for almost all of the four nights.

\section{The significance of the transits}

Because we will be dealing with potential unconfirmed transits or false positives, it is necessary to find a quantitative way to evaluate the significance of the individual transits observed. 
The observed transits are well sampled in the $V$ band, and the scattering is smaller than that of the OGLE transits. There are typically $N_{\text {tr }}=30-60$ points per transit in our data. VIMOS transits are well sampled, allowing us to measure accurate amplitudes as a difference between averages of the points outside and at the bottom of the transits. In the case of OGLE, the significance of the transits is in part judged by the number of transits detected (from a few to about 30). In the case of the present study, we compute the signal-to-noise of the single, well sampled transit. For a given photometric precision of a single measurement of $\sigma_{\mathrm{ph}}$ and a transit depth $A$, this signal-tonoise transit is $S / N=N_{\mathrm{tr}}^{1 / 2} A / \sigma_{\mathrm{ph}}$ (Gaudi 2005). For the monitored transits we find the range of $S / N$ to be 20-50 for typical $\sigma_{\mathrm{ph}}=0.004-0.005 \mathrm{mag}$. The error bars in the OGLE data are typically by $40-60 \%$ larger than those from VIMOS.

We also computed the significance of the transits considering the presence of correlated noise in the light curves, following the method described in Pont et al. (2006). Since we found that our light curves exhibit moderate red noise, the values of the $S / N$ reported below for different transits have been computed using this method.

It is also important to compute accurate mean times of transit as well as to evaluate the transit timing errors for studies of multiplicity in these systems, as it has been done in the case of OGLE-TR-111 by Díaz et al. (2008). The precision with which the mean transit time can be determined can be estimated as: $\delta_{\mathrm{tr}}=\sigma_{\mathrm{ph}} t_{\mathrm{tr}} /\left(2 A \sqrt{N_{\mathrm{tr}}}\right)$, where $t_{\mathrm{tr}}$ is the transit duration, and $N_{\mathrm{tr}}$ is the number of measurements within transit (Deeg et al. 2004).

\section{Full OGLE transits}

These objects allowed us to measure fundamental parameters of the systems by applying analytical and empirical models that mimic transiting light curves. In our work we used the method presented in Mandel \& Agol (2002). For error estimation we used the "rosary-bead" method fitting the light curves with the downhill-simplex algorithm (Bouchy et al. 2005; Southworth 2008; Winn et al. 2008). Below we discuss each of the observed objects.

\subsection{OGLE-TR-106}

In Fig. 3 we compare light curves for this object in the $I$ (OGLE) and $V$ (VIMOS) bands. The transit occurred at the beginning of night four as it was predicted. The shape of the event resembles a planetary transit, but it is not! Pont et al. (2005a), based on eight spectra obtained with the FLAMES instrument at the ESO VLT/UT2 telescope, show that this is an eclipsing binary, where the secondary is an $\mathrm{M}$ dwarf of the mass of only $0.116 \pm 0.021 M_{\odot}$. In Fig. 3 we also present our best fit to the VIMOS curve, using the linear limb-darkening coefficient for the $V$ band. This fit yields the ratios $r / R=0.145_{-0.006}^{+0.007}$, $a / R=13.3_{-2.1}^{+1.1}$, the impact parameter $b=a / R \cos i=0.35_{-0.35}^{+0.29}$ at the inclination $i \approx 90^{\circ}$. The limb-darkening law parameter was fixed to $u=0.6$. The estimated radius ratio is in agreement at $1 \sigma$ level with the value of $r / R=0.138 \pm 0.014$ derived from $R=1.31 \pm 0.09$ and $r=0.181 \pm 0.013$ (Pont et al. 2005a).

\subsection{OGLE-TR-109}

This is an extreme case among the transiting candidates found by the OGLE group because of the early spectral type of the star $(\mathrm{F} 0 \mathrm{~V})$, the low transit amplitude $\left(A_{I} \approx 0.008 \mathrm{mag}\right)$ and the
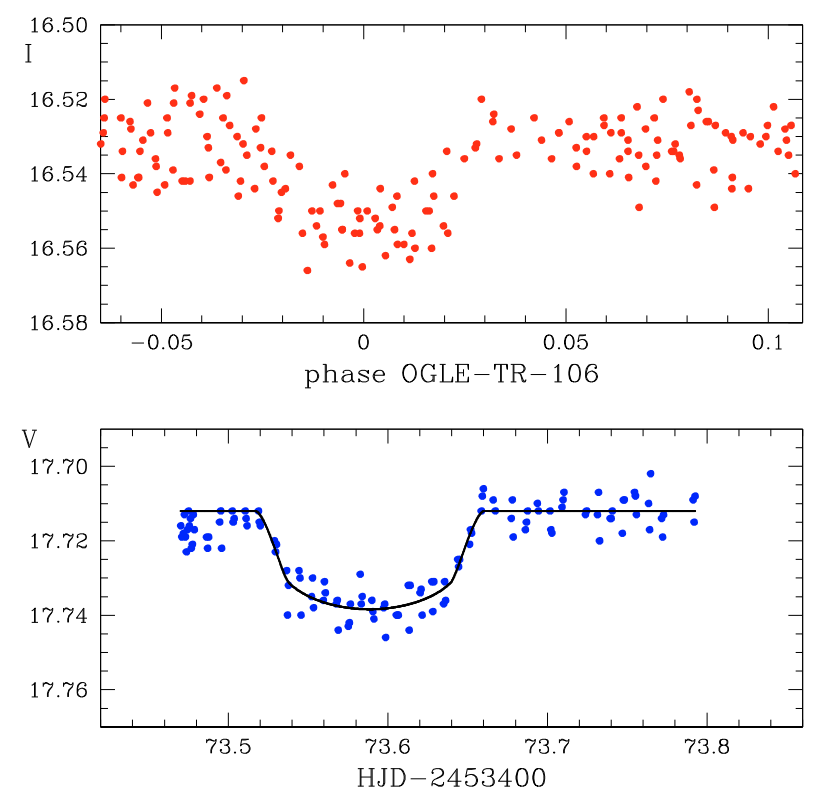

Fig. 3. Comparison of the light curves for OGLE-TR-106 from OGLE (top panel) and VIMOS (bottom panel). The magnitude scale and the time scale are the same for both panels. Fit to the VIMOS data is presented.
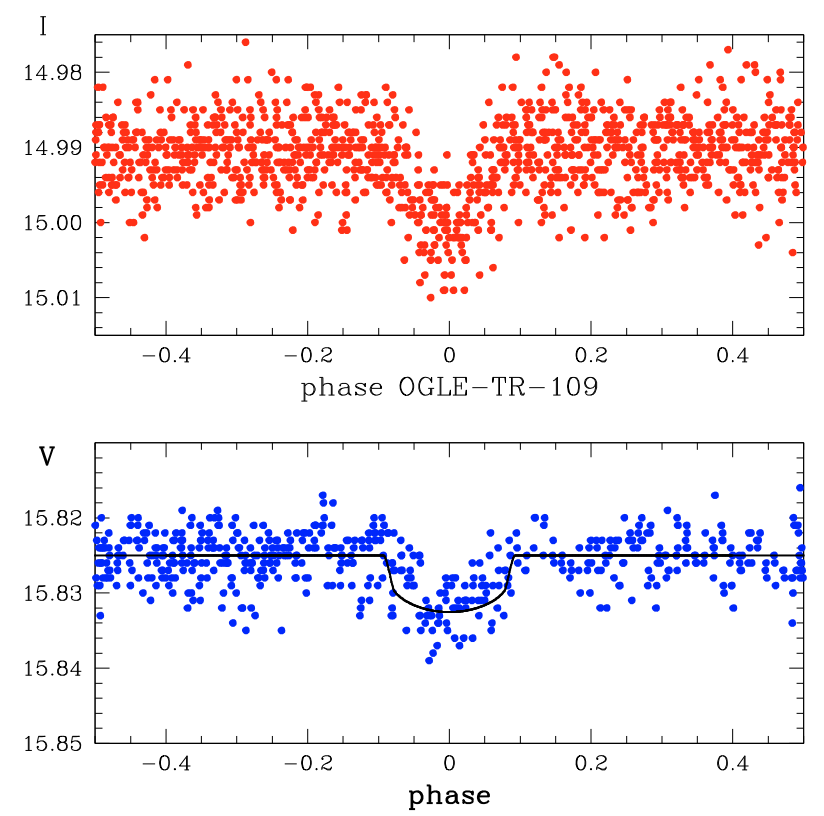

Fig. 4. Comparison of the light curves for OGLE-TR-109 from OGLE (top) and this work (bottom). The magnitude scale and the time scale are the same for both panels. The best fit to the VIMOS data is presented.

very short period $(P=0.589127 \mathrm{~d})$. An analysis of these photometric data by Fernández et al. (2006) and the analysis of highresolution spectroscopic data by Pont et al. (2005a) have left the nature of the object undetermined. Two scenarios are possible: OGLE-TR-109 is either a blend with a background eclipsing binary or a transiting planet. However, the latter possibility seems to be less likely because of the very short orbital period. Figure 4 shows both the OGLE and VIMOS light curves. In comparison to the results published in Fernández et al. (2006) the transit $S$ / $N$ is slightly better in this work (20 vs. 17), but still insufficient to resolve more details in the light curve. 
P. Pietrukowicz et al.: Millimagnitude photometry of 30 OGLE transits. V.
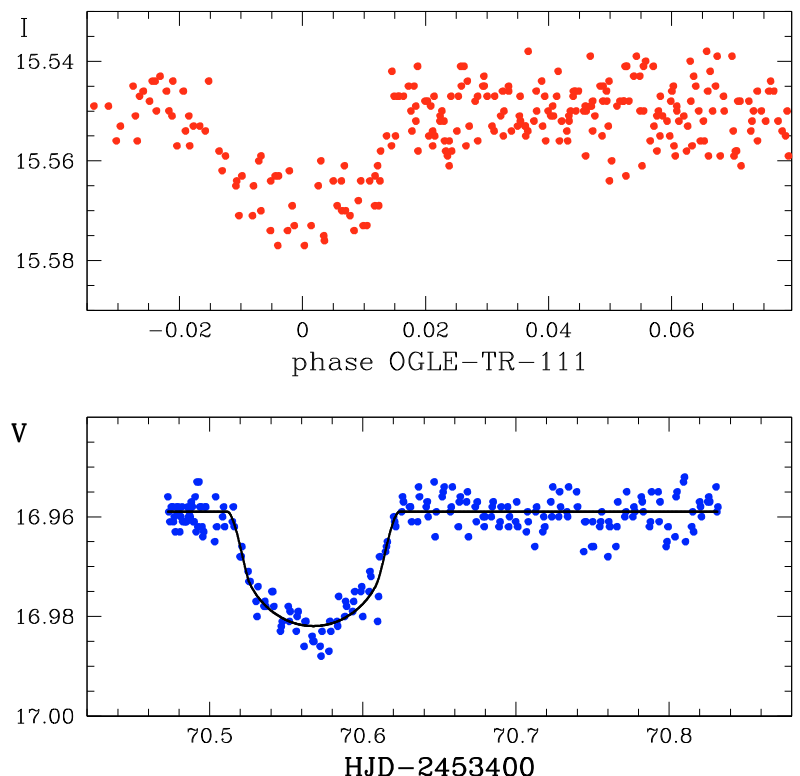

Fig. 5. Comparison of the light curves for OGLE-TR-111 from OGLE (top) and VIMOS (bottom). The magnitude scale and the time scale are the same for both panels. The figure also shows the best transit fit for this object.

\subsection{OGLE-TR-111}

This transit is caused by a hot Jupiter (Pont et al. 2004). It was analyzed in detail by Minniti et al. (2007). The VIMOS data allowed them to refine the planetary radius, obtaining $r=$ $1.01 \pm 0.06 R_{J}$. Our estimation of the radius ratios, $r / R=$ $0.1284_{-0.0033}^{+0.0066}$ and $a / R=12.31_{-1.56}^{+0.74}$, is in excellent agreement with the published values of $0.1245_{-0.0030}^{+0.0050}$ and $12.11_{-1.39}^{+1.00}$, respectively. Recently, Díaz et al. (2008) found possible period variations in this system which could be explained by the presence of a perturbing planet with the mass of the Earth in an exterior orbit. Figure 5 presents our best fit to the VIMOS data.

\subsection{OGLE-TR-113}

This is another planetary transit (Bouchy et al. 2004). It was expected to occur at the end of night two and it did. Díaz et al. (2007) used the VIMOS data to obtain new estimates for the orbit parameters, radius and mean density of the planet OGLETR-113b. The radius ratios we have found are almost identical to the values they obtained: $r / R=0.1451_{-0.0022}^{+0.0064}$ vs. $0.1455 \pm$ 0.0083 , and $a / R=6.49_{-0.66}^{+0.10}$ vs. $6.48 \pm 0.09$. The light curves and the fit are shown in Fig. 6. Our new period estimation, $P=1.4324772(12) \mathrm{d}$, is in excellent agreement with the value of 1.4324757(13) d derived by Gillon et al. (2006) on the base of NTT/SUSI2 data taken almost at the same time (on 2005 Apr. 3 and 13).

\subsection{OGLE-TR-159}

This object is located in the field of OGLE-TR-167. A transit occurred in the middle of night two, as it was expected from OGLE ephemeris. The transit duration $t_{\mathrm{tr}}$ was approximately $3.2 \mathrm{~h}$ with about 45 data points $\left(\delta_{\mathrm{tr}}=1.3 \mathrm{~min}\right)$. We measured an amplitude $A_{V}=0.045 \pm 0.006$ mag with a transit signal-to-noise of $S / N \approx 50$, in agreement with $I$-band measurements at $1 \sigma$ level: $A_{I}=0.040 \pm 0.015 \mathrm{mag}$. The transit portion of the light curve
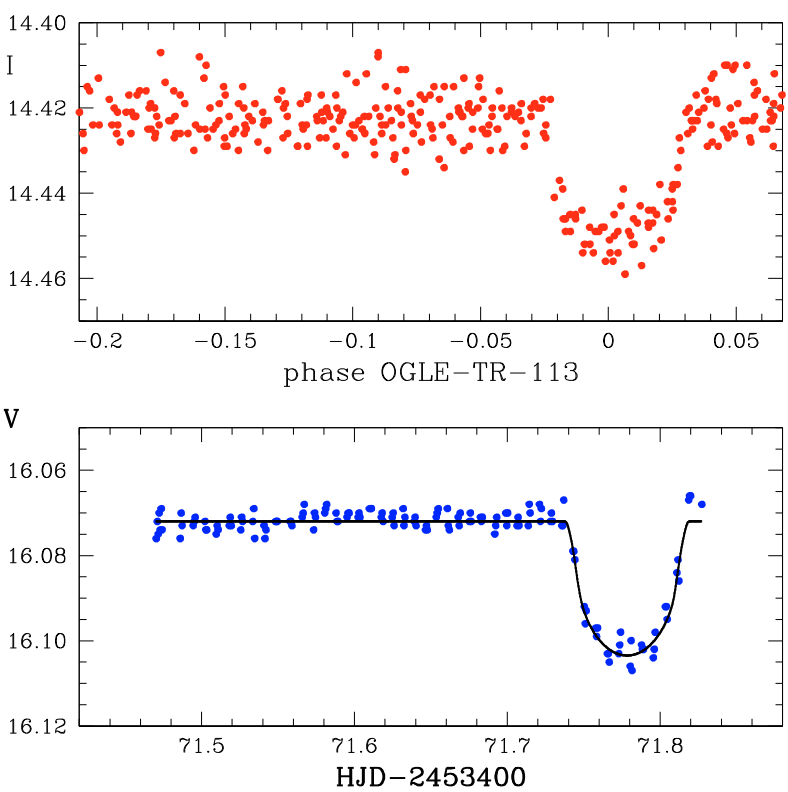

Fig. 6. Comparison of the light curves for OGLE-TR-113 from OGLE in the $I$ band (top panel) and VIMOS in the $V$ band (bottom panel). The magnitude scale and the time scale are the same for both panels. The solid line presents the best fit for this transit.
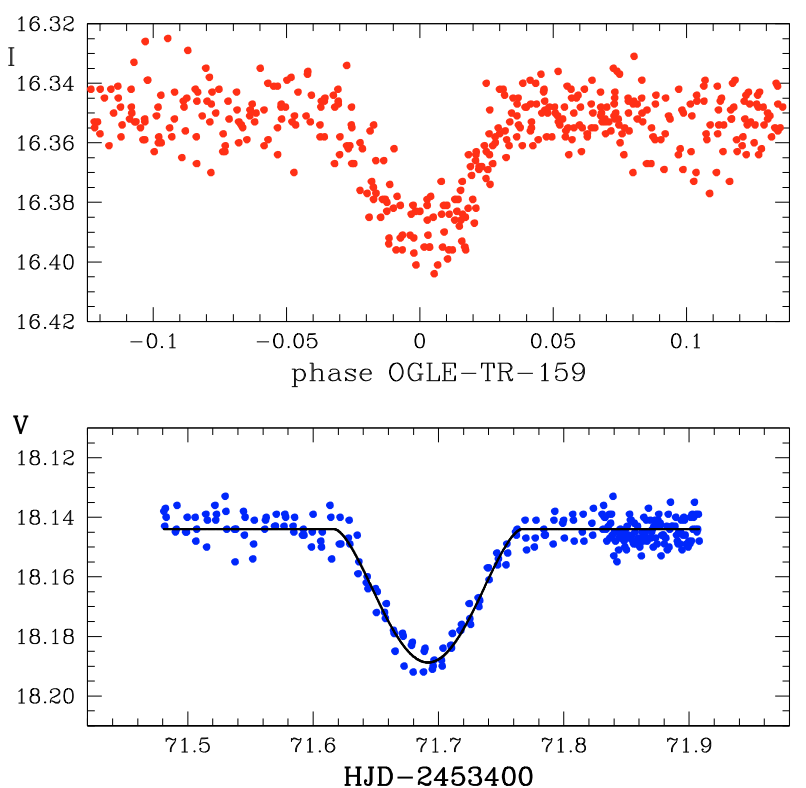

Fig. 7. Comparison of the light curves for OGLE-TR-159 from OGLE (top) and this work (bottom). The magnitude scale and the time scale are the same for both panels. Fit to the VIMOS data is shown.

does not show a flat bottom (see Fig. 7), indicating a large impact parameter for this system. Following Mandel \& Agol (2002), we obtained $r / R=0.41_{-0.20}^{+0.03}$ and inclination $i=74^{\circ}$. We conclude that OGLE-TR-159 is very likely an eclipsing binary.

\subsection{OGLE-TR-170}

The transit OGLE-TR-170 occurred in the middle of night three and lasted for $t_{\text {tr }} \approx 4.3 \mathrm{~h}$ (see Fig. 8). There are more than 70 photometric points $\left(\delta_{\mathrm{tr}}=1.8 \mathrm{~min}\right)$ within the transit. We measured an amplitude of $A_{V}=0.036 \pm 0.006$ mag with a transit signalto-noise of $S / N \approx 50$, again in agreement at $1 \sigma$ level with the 

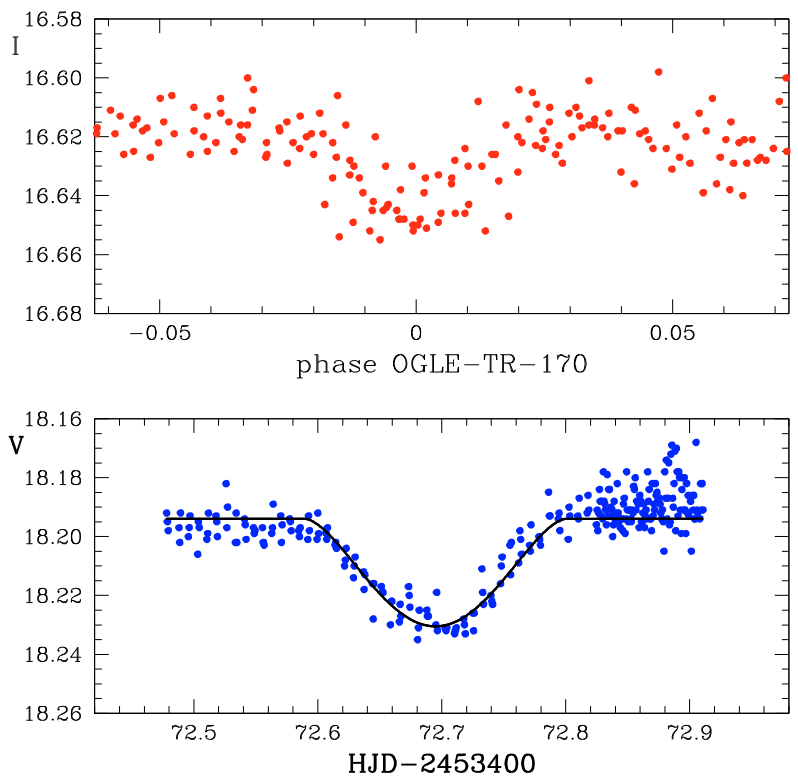

Fig. 8. Comparison of the light curves for OGLE-TR-170 from OGLE (top) and VIMOS (bottom). The magnitude scale and the time scale are the same for both panels. The lower panel shows a fit of the event.

$I$-band measurements: $A_{I}=0.030 \pm 0.015 \mathrm{mag}$. As for OGLETR-159, this transit does not show a flat bottom. It is very likely that OGLE-TR-170 is an eclipsing system observed during a grazing eclipse.

\subsection{OGLE-TR-171}

This transit occurred at the beginning of night three lasting about $2.5 \mathrm{~h}$. We measured an amplitude $A_{V}=0.032 \pm 0.009$ mag with a transit signal-to-noise of $S / N \approx 22$, which gave similar values as in the $I$ band: $A_{I}=0.038 \pm 0.015, t_{\mathrm{tr}}=2.5 \mathrm{~h}$. The transit seems to be asymmetric (Fig. 9). In both light curves, OGLE and VIMOS, the minimum occurred earlier than the central moment of the event. The asymmetry cannot be caused by a transiting planet, but could indicate a matter flow between the components or the presence of a disk around one of the stars.

In Table 3 we summarize geometrical parameters obtained for six out of seven fully observed OGLE transits. In the case of OGLE-TR-159 and OGLE-TR-170, for which V-shape eclipses were detected, the obtained parameter distributions are bimodal, and therefore the given numbers are not very representative of the distributions. In the table we report median values at $68 \%$ confidence level.

\section{Partial OGLE transits}

\subsection{OGLE-TR-86}

This transit occurred at the end of night one as it was expected from the OGLE data. We got only the ingress phase (see Fig. 10), so we could not measure the flatness of the light curve during the event.

\subsection{OGLE-TR-91}

The transit OGLE-TR-91 was expected in the middle of night one, but it occurred at the beginning of that night. The transit portion of the light curve (Fig. 11) shows a flat bottom, indicating a relatively small impact parameter for this system. We measured
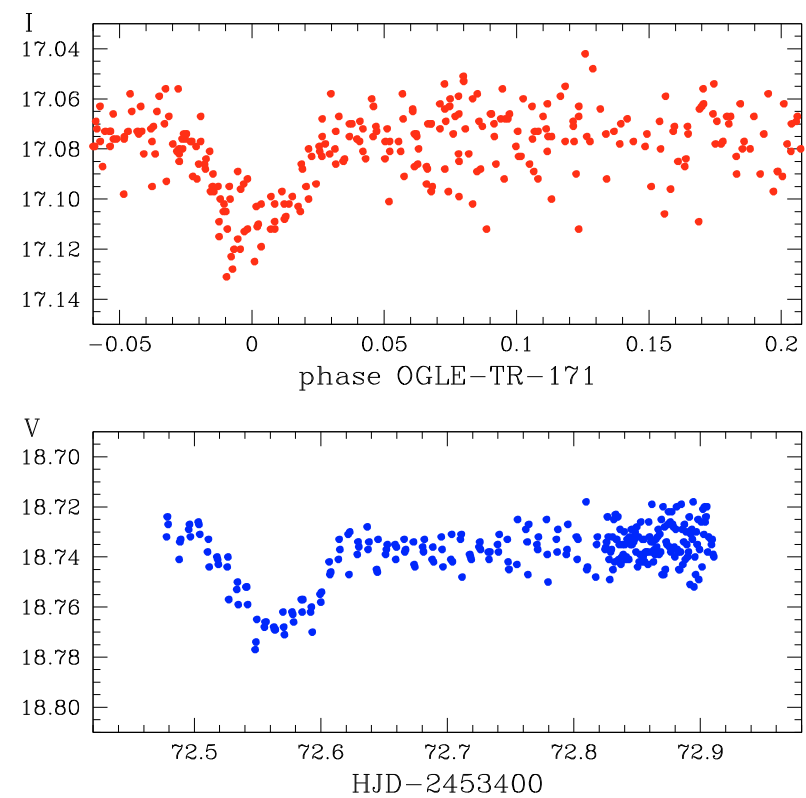

Fig. 9. Comparison of the light curves for OGLE-TR-171 from OGLE (top) and VIMOS data (bottom). The magnitude scale and the time scale are the same for both panels. The shape of the transit is clearly asymmetric, and therefore no fit is given for this object.

Table 3. Geometrical parameters determined for six systems.

\begin{tabular}{ccccc}
\hline \hline Transit & $r / R$ & $a / R$ & $b$ & $u$ \\
\hline OGLE-TR-106 & $0.1454_{-0.0060}^{+0.0071}$ & $13.3_{-2.1}^{+1.1}$ & $0.35_{-0.35}^{+0.29}$ & 0.6 \\
OGLE-TR-109 & $0.0771_{-0.0029}^{+0.0037}$ & $1.88_{-0.25}^{+0.12}$ & $0.17_{-0.16}^{+0.48}$ & $0.597^{*}$ \\
OGLE-TR-111 & $0.1284_{-0.0033}^{+0.0066}$ & $12.31_{-1.56}^{+0.74}$ & $0.31_{-0.31}^{+0.25}$ & $0.768^{*}$ \\
OGLE-TR-113 & $0.1451_{-0.0022}^{+0.0064}$ & $6.49_{-0.66}^{+0.10}$ & $0.11_{-0.09}^{+0.35}$ & $0.780^{*}$ \\
OGLE-TR-159 & $0.41_{-0.20}^{+0.03}$ & $4.18_{-0.15}^{+0.24}$ & $1.11_{-0.15}^{+0.05}$ & 0.6 \\
OGLE-TR-170 & $0.23_{-0.05}^{+0.19}$ & $2.96_{-0.10}^{+0.19}$ & $0.91_{-0.12}^{+0.26}$ & 0.6 \\
\hline
\end{tabular}

In the columns we provide: ratio of the radii of the components $r / R$, ratio of the radius of the orbit of the secondary to the radius of the primary, impact parameter $b$, and linear limb-darkening coefficient $u$. For objects OGLE-TR-109, OGLE-TR-111, and OGLE-TR-113 the limbdarkening coefficient was taken from Claret (2000).

an amplitude of $A_{V}=0.037 \pm 0.004 \mathrm{mag}$, in agreement with OGLE measurements: $A_{I}=0.043 \pm 0.015 \mathrm{mag}$. However, the trapezium-like shape of the event with long ingress and egress rather rules out a planetary nature of the transit. A light curve modulation, probably due to ellipsoidal variability, is also seen.

\subsection{OGLE-TR-110}

This object is located in the field F113. We observed only the egress of the transit on night two (Fig. 12). Good agreement with the prediction time indicates good estimation of the period from OGLE data. The slow egress (0.03 mag in $1.5 \mathrm{~h})$ clearly rules out a planetary nature of the event. This was confirmed by Pont et al. (2005a), who found a large radial velocity difference in two sets of spectral lines, like in a binary.

\subsection{OGLE-TR-167}

This transit occurred at the end of night two as was expected from the OGLE data. As for OGLE-TR-86, we only got the 
P. Pietrukowicz et al.: Millimagnitude photometry of 30 OGLE transits. V.
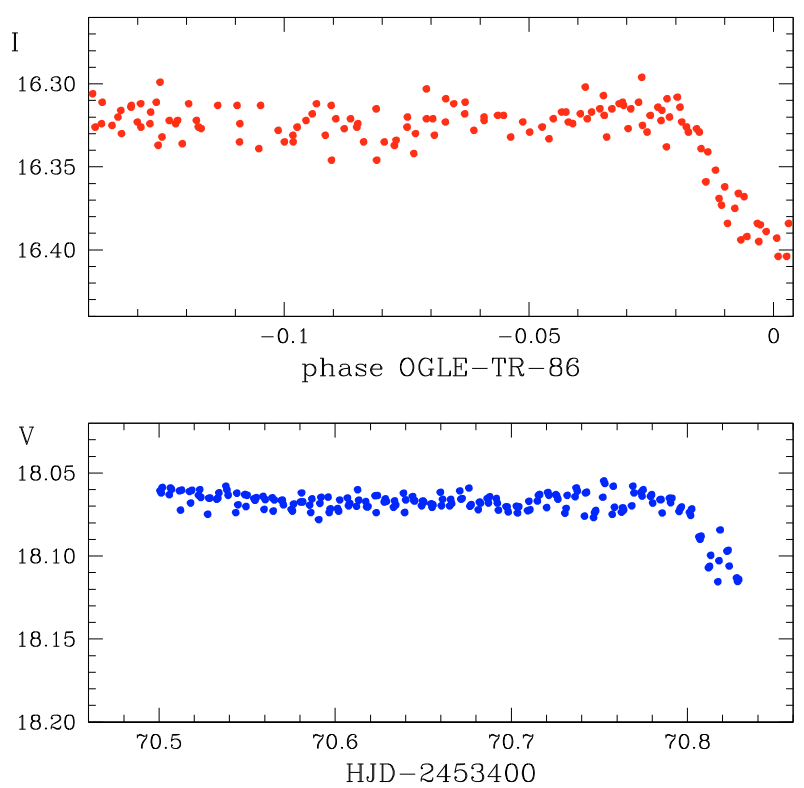

Fig. 10. Comparison of the light curves for OGLE-TR-86 from OGLE (top) and this work (bottom). The magnitude scale and the time scale are the same for both panels.
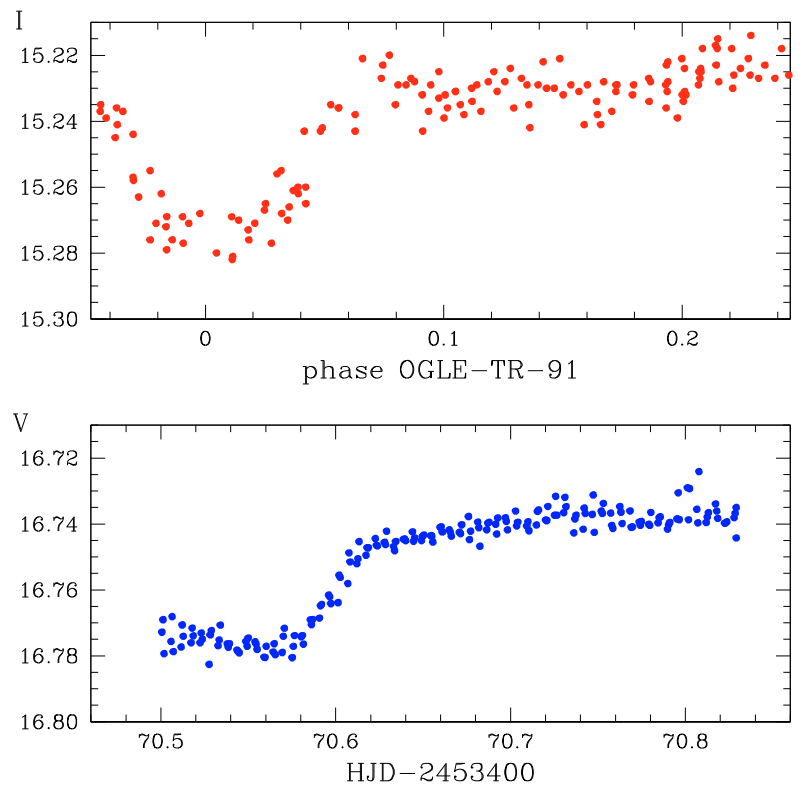

Fig. 11. Comparison of the light curves for OGLE-TR-91 from OGLE in the $I$ band (top) and VIMOS in the $V$ band (bottom). The magnitude scale and the time scale are the same for both panels.

beginning of the transit of OGLE-TR-167 (Fig. 13), so we couldn't measure the flatness of the light curve during the event.

For all partial transits but OGLE-TR-91 only $V$-band amplitude lower limits could be measured. For the object OGLETR-91, which is the only partial transit with a flat bottom, we estimated the central moment of the event. For the other three objects their periods were improved using either ingress or egress moments. From the shape of the observed partial transits we conclude that none of them was caused by a planet.

In Table 4 we summarize photometric data of eleven objects with observed transits. The measured $V$-band amplitudes of full transits are generally larger than the amplitudes measured in the $I$ band (see Fig. 14). This agrees with the fact that stellar limb darkening in the $V$ band should be shallower at the edges but
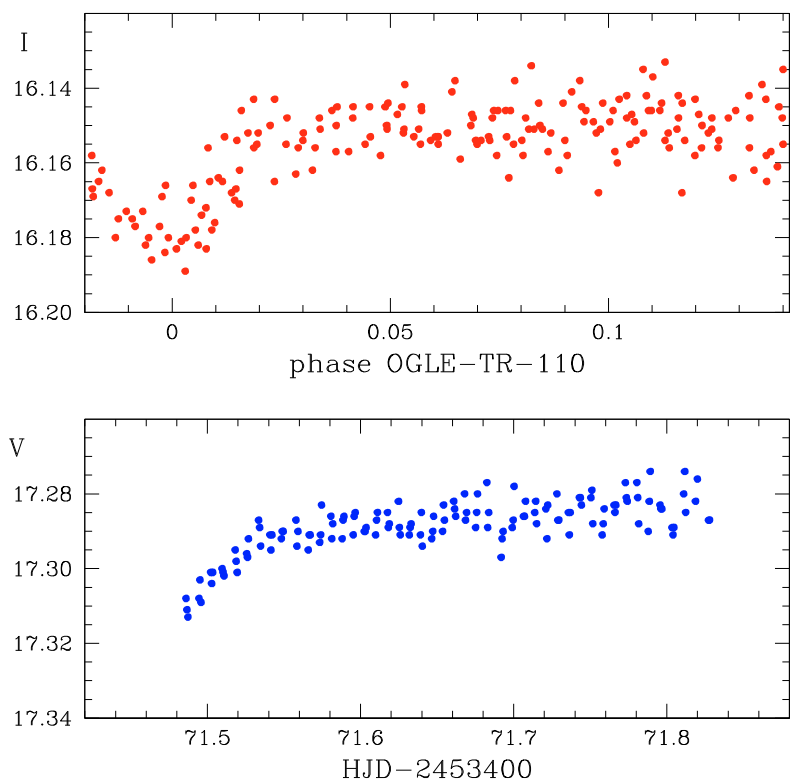

Fig. 12. Comparison of the light curves for OGLE-TR-110 from OGLE (top) and this work (bottom). The magnitude scale and the time scale are the same for both panels.
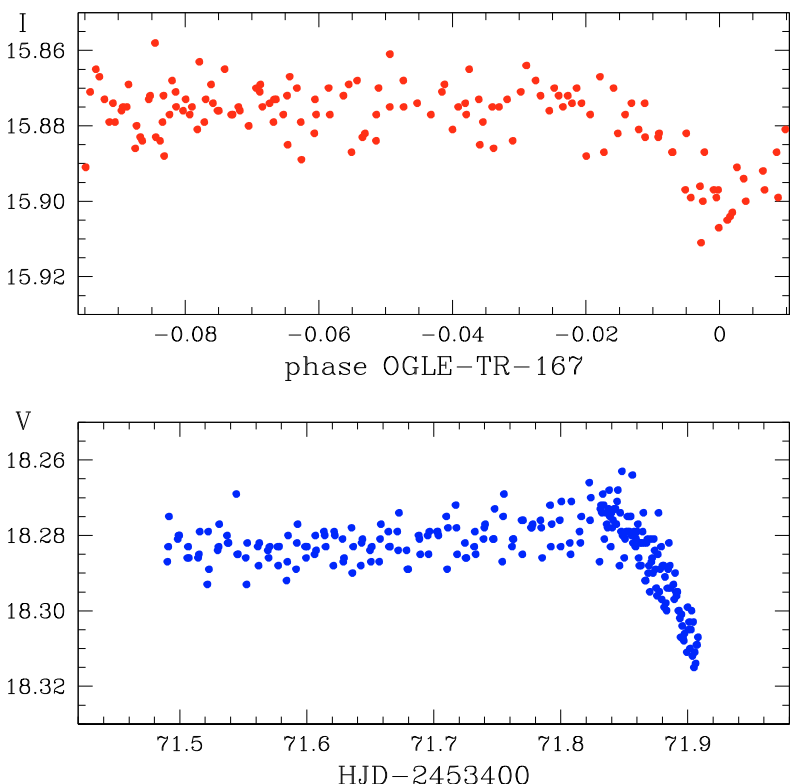

Fig. 13. Comparison of the light curves for OGLE-TR-167 from OGLE (top) and this work (bottom). The magnitude scale and the time scale are the same for both panels.

deeper by $10-20 \%$ in the central parts (Claret \& Hautschildt 2003).

\section{Absent OGLE transits}

\subsection{OGLE-TR-82}

This star lies in the field F86, which was monitored for only eight hours. A transit was predicted to occur at the end of night one, but the observations of the field were finished about two hours before dawn, and no event could be recorded. The flat portion of the light curve (see Fig. 15) was measured with a photometric precision of $0.003 \mathrm{mag}$, allowing us to detect a sinusoidal variation of a full amplitude of $0.0035 \mathrm{mag}$ and a period of $\sim 0.23 \mathrm{~d}$, 
Table 4. Photometric data on the OGLE objects for which either full or partial transits were observed with VIMOS.

\begin{tabular}{|c|c|c|c|c|c|c|c|c|c|c|c|c|}
\hline $\begin{array}{l}\text { OGLE } \\
\text { transit }\end{array}$ & $\begin{array}{c}I \\
{[\mathrm{mag}]}\end{array}$ & $\begin{array}{c}A_{I} \\
{[\mathrm{mag}]}\end{array}$ & $\begin{array}{c}\sigma_{I} \\
{[\mathrm{mag}]}\end{array}$ & $\begin{array}{c}V \\
{[\mathrm{mag}]}\end{array}$ & $\begin{array}{c}A_{V} \\
{[\mathrm{mag}]}\end{array}$ & $\begin{array}{c}\sigma_{V} \\
{[\mathrm{mag}]}\end{array}$ & $\mathrm{HJD}_{0}-2453400$ & $\begin{array}{c}t_{\mathrm{tr}} \\
{[\mathrm{h}: \mathrm{m}]}\end{array}$ & $N_{\text {tr }}$ & $\begin{array}{c}\delta_{\mathrm{tr}} \\
{[\mathrm{m}]}\end{array}$ & $E$ & $\begin{array}{c}\text { New period } \\
\text { [d] }\end{array}$ \\
\hline TR-106 & 16.53 & 0.022 & 0.009 & 17.71 & 0.026 & 0.005 & 73.5885(5) & $3: 00$ & 50 & 1.8 & 453 & $2.535994(2)$ \\
\hline TR-109 & 14.99 & 0.008 & 0.004 & 15.82 & 0.008 & 0.002 & $72.540(1)$ & 2:05 & 40 & 2.3 & 1950 & $0.5891262(8)$ \\
\hline TR-111 & 15.55 & 0.019 & 0.005 & 16.96 & 0.023 & 0.003 & $70.5676(5)$ & $2: 40$ & 60 & 1.1 & 284 & $4.014510(4)^{*}$ \\
\hline TR-113 & 14.42 & 0.030 & 0.003 & 16.07 & 0.031 & 0.003 & $71.7782(5)$ & 1:55 & 30 & 0.7 & 801 & $1.4324772(12)$ \\
\hline TR-159 & 16.35 & 0.038 & 0.008 & 18.14 & 0.045 & 0.006 & $71.6917(5)$ & 3:10 & 45 & 1.3 & 364 & $2.126770(3)$ \\
\hline TR-170 & 16.62 & 0.026 & 0.008 & 18.19 & 0.036 & 0.006 & $72.6949(5)$ & $4: 15$ & 70 & 1.8 & 187 & $4.136697(5)$ \\
\hline TR-171 & 17.07 & 0.038 & 0.012 & 18.73 & 0.032 & 0.009 & $72.562(1)$ & $2: 30$ & 40 & 2.4 & 371 & $2.091819(4)$ \\
\hline TR-86 & 16.32 & 0.065 & 0.008 & 18.07 & $>0.050$ & 0.006 & $70.85(1)$ & - & - & - & 413 & $2.77702(2)$ \\
\hline TR-91 & 15.23 & 0.043 & 0.004 & 16.74 & 0.035 & 0.005 & $70.54(1)$ & - & - & - & 726 & $1.57883(2)$ \\
\hline TR-110 & 16.15 & 0.026 & 0.007 & 17.28 & $>0.025$ & 0.007 & $71.45(1)$ & - & - & - & 402 & $2.84852(2)$ \\
\hline TR-167 & 15.88 & 0.022 & 0.006 & 18.28 & $>0.025$ & 0.008 & $71.95(1)$ & - & - & - & 147 & $5.26066(7)$ \\
\hline
\end{tabular}

The last five columns give respectively: central moment in Heliocentric Julian Days, transit duration $t_{\mathrm{tr}}$, number of measurements within transit $N_{\text {tr }}$, mean transit time precision $\delta_{\text {tr }}$ (see text), number of epochs $E$ that have passed since OGLE observations, new period estimation. Note the dramatic increase in precision of the periods in comparison to the values estimated by OGLE (Table 2). Also note that period variations in the object OGLE-TR-111 (marked with *) have been recently reported by Díaz et al. (2008).

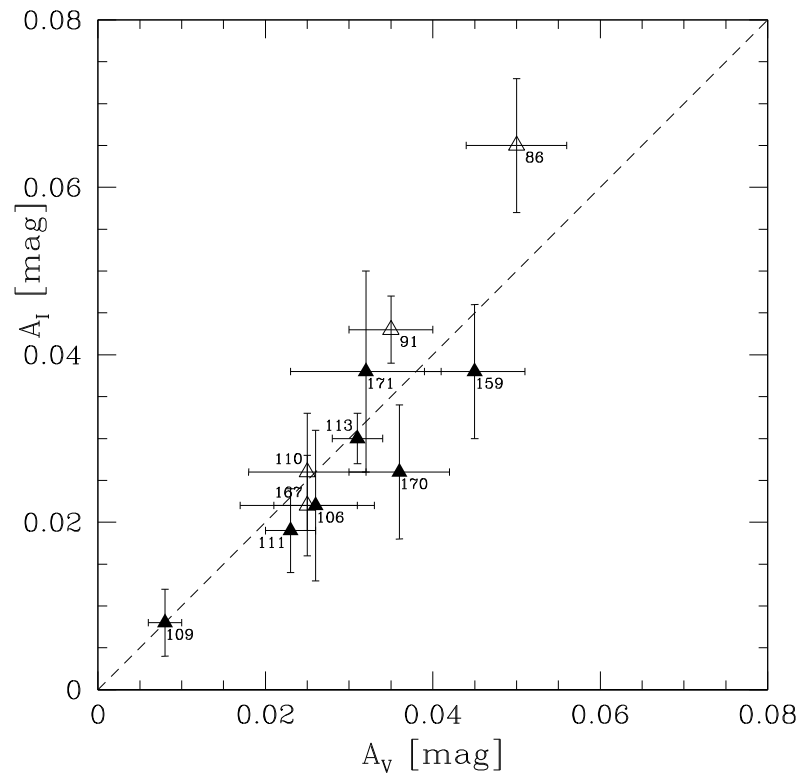

Fig. 14. $I$-band amplitudes vs. $V$-band amplitudes of the transits. Fully and partially observed transits are marked with full and open triangles, respectively.

which is different from the transiting period of $P=0.76416 \mathrm{~d}$. Recently Hoyer et al. (2007) have shown that the system is a main-sequence binary blended with a background red giant. The variability may come from the blend.

\subsection{OGLE-TR-84}

This object is located in the field F86. According to Pont et al. (2005a) it is likely an eclipsing system. An eclipse was expected at the beginning of night one, but it was not detected.

\subsection{OGLE-TR-85}

Probably this is a triple system (Pont et al. 2005a). In this case we expected to see a transit at the beginning of night one, but nothing was observed.
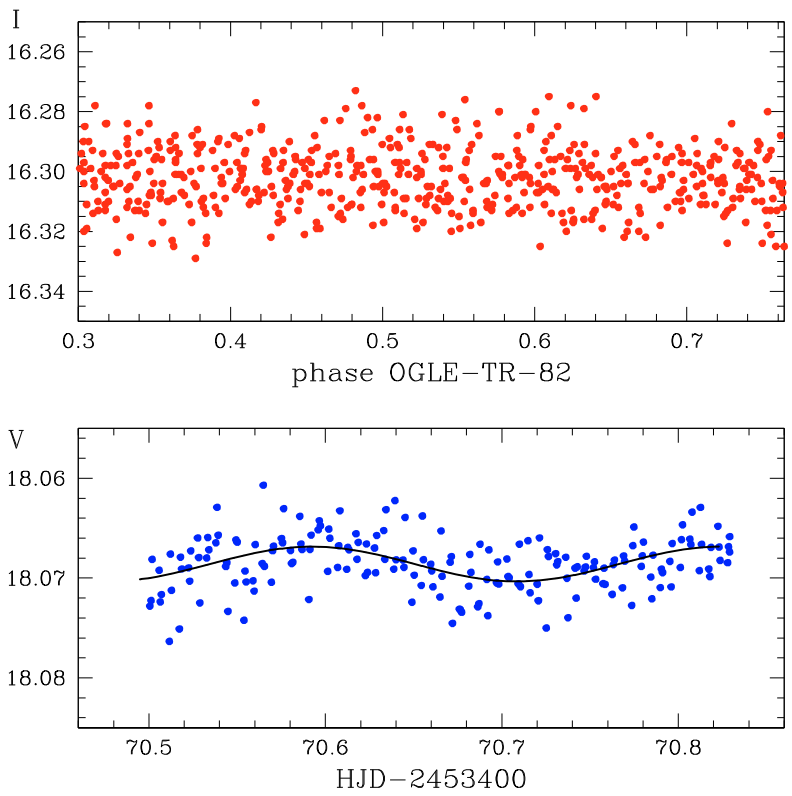

Fig. 15. Comparison of the light curves for OGLE-TR-82 from OGLE (top) and VIMOS (bottom). A sinusoidal fit to the VIMOS data is presented.

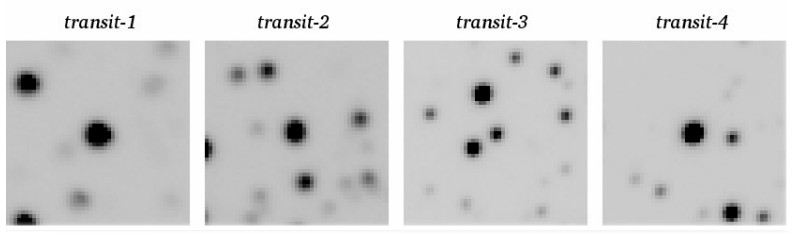

Fig. 16. Finding charts for the new transit candidates. North is up and East to the left. The field of view is $10^{\prime \prime}$ on a side. The transiting stars lie exactly in the centers of the charts.

\subsection{OGLE-TR-108}

This star is located in F113, field which was observed during all four nights. Our ephemerides for OGLE-TR-108 predicted a transit in the middle of night three, and a secondary transit for night one, but we did not detect anything. 
P. Pietrukowicz et al.: Millimagnitude photometry of 30 OGLE transits. V.
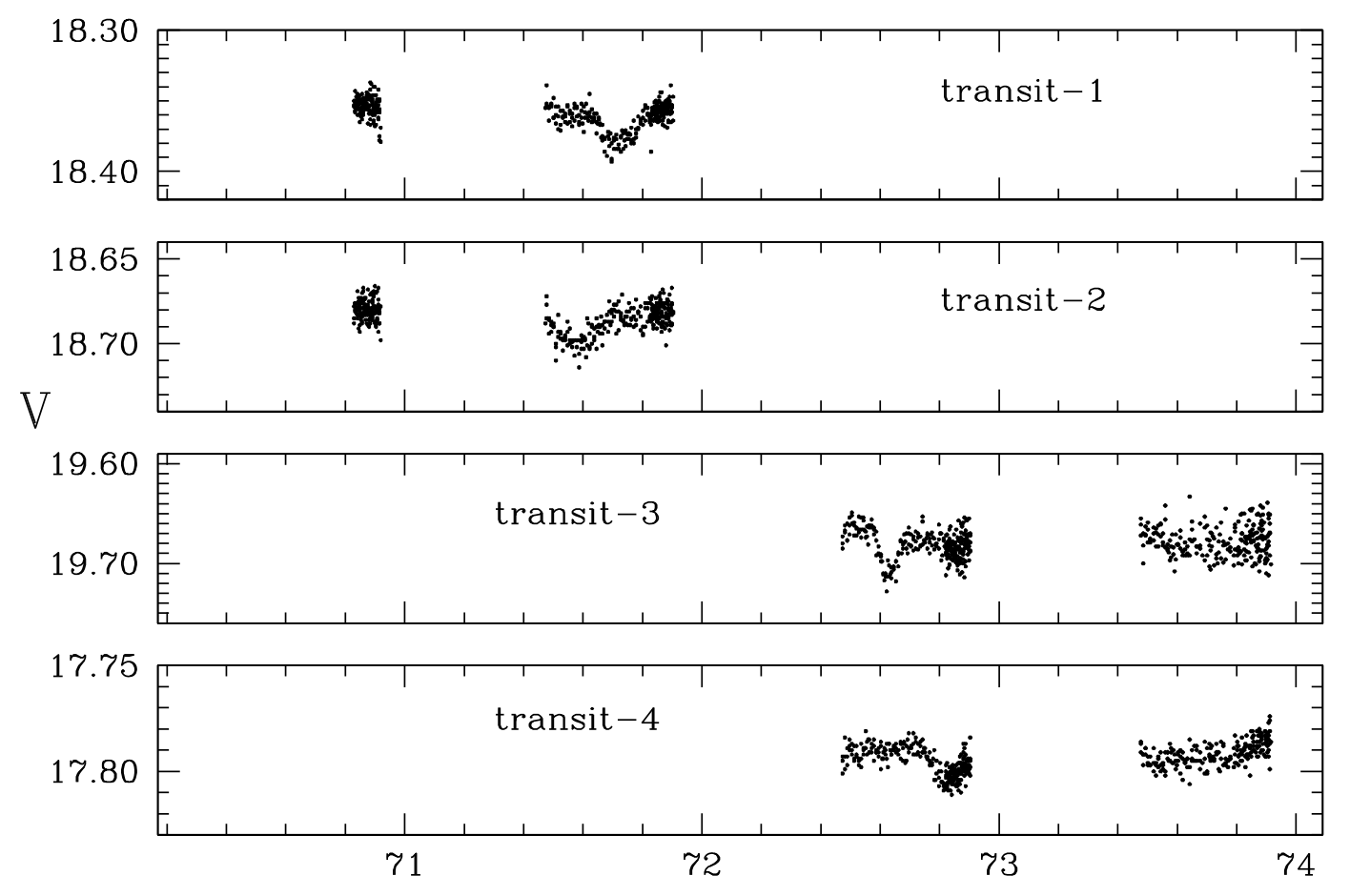

HJD - 2453400

Fig. 17. New transit candidates found in the VIMOS data.

Table 5. Photometric information on four new transit candidates.

\begin{tabular}{ccccccc}
\hline \hline New candidate & $\begin{array}{c}\text { RA(2000.0) } \\
{[\mathrm{h}: \mathrm{m}: \mathrm{s}]}\end{array}$ & $\begin{array}{c}\text { Dec(2000.0) } \\
{\left[{ }^{\circ}::^{\prime \prime}\right]}\end{array}$ & $\begin{array}{c}V \\
{[\mathrm{mag}]}\end{array}$ & $\begin{array}{c}A_{V} \\
{[\mathrm{mag}]}\end{array}$ & $\mathrm{HJD}_{0}-2453400$ & $\begin{array}{c}\text { Duration } \\
{[\mathrm{h}: \mathrm{m}]}\end{array}$ \\
\hline transit-1 & $13: 30: 21.33$ & $-64: 05: 39.3$ & 18.36 & 0.025 & 71.723 & $3: 40$ \\
transit-2 & $13: 30: 20.73$ & $-64: 07: 50.9$ & 18.68 & 0.025 & 71.573 & $4: 50$ \\
transit-3 & $13: 14: 45.62$ & $-64: 40: 28.2$ & 19.65 & 0.040 & 72.633 & $2: 10$ \\
transit-4 & $13: 13: 34.77$ & $-64: 50: 50.9$ & 17.79 & 0.020 & 72.833 & $3: 40$ \\
\hline
\end{tabular}

\subsection{OGLE-TR-114}

A spectroscopic follow-up made by Pont et al. (2005a) showed that this object is a triple system. A transit was expected at the end of night four, but due to bad seeing there is no clear evidence for the event.

\subsection{OGLE-TR-162}

This object can be found in the field F167. In this case we hoped to observe a transit in the middle of night two, but nothing was detected.

\subsection{OGLE-TR-172}

The star is located in the VIMOS field F170. A transit expected to occur in the middle of night four was not observed.

It is very likely that the seven transits mentioned above were not observed due to two facts: a short VIMOS observational run and lost of ephemerides for the objects. This is clearly seen in the case of the transits from the field F86 which was observed only for $8 \mathrm{~h}$. The time interval between the OGLE and VIMOS observations was approximately three years. Exact time uncertainties in the OGLE data are not given, but we assess them to be below $0.0005 \mathrm{~d}$. After the three years the errors of the expected moments of the transits could have accumulated to a significant fraction of the orbital period.

\section{New candidates}

We have also looked for new transit candidates in the VIMOS data. This has been done in the framework of our variable search presented in Pietrukowicz et al. (2009). Finding charts and light curves of four new candidates are shown in Figs. 16 and 17 respectively. Table 5 gives the most important observational facts on the transits. The first two objects are located in the field F167 and the other two in the field F170. These are rather faint stars with $17.8<V<19.7$ mag. The transits have amplitudes between 0.02 and $0.04 \mathrm{mag}$ in $V$ and durations from about 2 to $5 \mathrm{~h}$. All events were detected only once during our VIMOS run, therefore there is no information on periods. We also note that for the transits 1-3 no events were detected in the OGLE-III data. The transit-4, due to its depth and relatively long duration, is potentially the best candidate for hosting a planet. This object lies outside OGLE-III fields and is a good target for future surveys.

\section{Summary}

$V$-band images from VLT/VIMOS were used to obtain light curves of extrasolar planetary transits: OGLE-TR-111, OGLE-TR-113 and candidate planetary transits: OGLE-TR109, OGLE-TR-159, OGLE-TR-167, OGLE-TR-170, OGLETR-171. With difference imaging photometry we were able to achieve millimagnitude errors in the individual data points. The 
following seven OGLE transits were recorded as full events: $106,109,111,113,159,170,171$. Four transits were detected as partial events: $86,91,110,167$. All full and partial transits but OGLE-TR-91 and OGLE-TR-109 were observed at the predicted transit times. No transits were recorded for 19 objects.

Based on the shape of the obtained light curves and some results from spectroscopic follow-up studies we show that the objects OGLE-TR-111 and OGLE-TR-113 are probably the only OGLE stars in the sample to host planets.

In the paper we also report on four new transiting candidates we have found in the VIMOS data. One of the events, transit-4, with the duration time of about $3.7 \mathrm{~h}$ and the amplitude of about $0.02 \mathrm{mag}$, is a particularly good candidate for a planetary transit. Faintness of the object (17.8 mag in $V$ ) may severely hamper spectroscopic verification. However, all four candidates require photometric follow-up studies to look for their periodic nature first.

Acknowledgements. P.P., D.M., J.M.F., G.P., M.Z., M.T.R., W.G., M.H. are supported by FONDAP Center for Astrophysics No. 15010003 and the BASAL Center for Astrophysics and Associated Technologies. P.P. was also supported by the Foundation for Polish Science through program MISTRZ and the Polish Ministry of Science and Higher Education through the grant N N203 301335. M.Z. acknowledges support by Proyecto FONDECYT Regular No. 1085278. The OGLE project is partially supported by the Polish MNiSW grant N20303032/4275. D.M. also thanks the John Simon Guggenheim Foundation. A.U. acknowledges support from the grant "Subsydium Profesorskie" from the Foundation for Polish Science. We thank the ESO staff at Paranal Observatory.

\section{References}

Alard, C., \& Lupton, J. 1998, ApJ, 503, 325

Baraffe, I., Chabrier, G., Barman, T. S., et al. 2003, A\&A, 402, 701

Bouchy, F., Pont, F., Santos, N. C., et al. 2004, A\&A, 421, L13
Bouchy, F., Pont, F., Melo, C., et al. 2005, A\&A, 431, 1105 Burrows, A., Ram, R. S., Bernath, P., et al. 2002, ApJ, 577, 986 Charbonneau, D., Brown, T. M., Latham, D. W., et al. 2000, ApJ, 529, L45 Claret, A. 2000, A\&A, 363, 1081

Claret, A., \& Hautschildt, P. 2003, A\&A, 412, 241

Deeg, H. J., Alonso, R., Belmonte, J. A., et al. 2004, PASP, 116, 985 Díaz, R. F., Ramírez, S., Fernández, J. M., et al. 2007, ApJ, 660, 850 Díaz, R. F., Rojo, P., Melita, M., et al. 2008, ApJ, 682, L49 Drake, A. J. 2003, ApJ, 589, 1020

Fernández, J. M., Minniti, D., Pietrzyński, G., et al. 2006, ApJ, 647, 587 Gaudi, S. A. 2005, ApJ, 628, L73

Gillon, M., Pont, F., Moutou, C., et al. 2006, A\&A, 459, 249

Henry, G. W., Marcy, G. W., Butler, R. P., et al. 2000, ApJ, 529, L41 Hoyer, S., Ramírez Alegría, S., Ivanov, V. D., et al. 2007, ApJ, 669, 1345

Konacki, M., Torres, G., Jha, S., et al. 2003, Nature, 421, 507

Konacki, M., Torres, G., Sasselov, D. D., et al. 2004, ApJ, 609, L37

Konacki, M., Torres, G., Sasselov, D. D., et al. 2005, ApJ, 624, 372

LeFevre, O., Saisse, M., Mancini, D., et al. 2003, SPIE, 4841, 1670

Mandel, K., \& Agol, E. 2002, ApJ, 580, L171

Mayor, M., \& Queloz, D. 1995, Nature, 378, 355

Minniti, D., Fernández, J. M., Díaz, R. F., et al. 2007, ApJ, 660, 858

Moutou, C., Pont, F., Bouchy, F., et al. 2004, A\&A, 424, L31

Pepper, J., \& Gaudi, S. A. 2005, ApJ, 631, 581

Pietrukowicz, P., Minniti, D., Fernández, J. M., et al. 2009, A\&A, 503, 651

Pont, F., Bouchy, F., Queloz, D., et al. 2004, A\&A, 426, L15

Pont, F., Bouchy, F., Melo, C., et al. 2005a, A\&A, 438, 1123

Pont, F., Melo, C. H. F., Bouchy, F., et al. 2005b, A\&A, 433, L21

Pont, F., Zucker, S., \& Queloz, D. 2006, MNRAS, 373, 231

Pont, F., Tamuz, O., Udalski, A., et al. 2008, A\&A, 487, 749

Sirko, E., \& Paczyński, B. 2003, ApJ, 592, 1217

Southworth, J. 2008, MNRAS, 386, 1644

Stetson, P. B. 1987, PASP, 99, 191

Udalski, A., Paczyński, B., Żebruń, K., et al. 2002a, Acta Astron., 52, 1

Udalski, A., Szewczyk, O., Żebruń, K., et al. 2002b, Acta Astron., 52, 317

Udalski, A., Pietrzyński, G., Szymański, M., et al. 2003, Acta Astron., 53, 133

Udalski, A., Pont, F., Naef., D., et al. 2008, A\&A, 482, 299

Winn, J. N., Holman, M. J., Torres, G., et al. 2008, ApJ, 683, 1076

Woźniak, P. R. 2000, Acta Astron., 50, 421 